\title{
Effet de la pression du mélange équimolaire oxygène-protoxyde d'azote (MEOPA) sur l'efficacité de la sédation : étude transversale sur 71 enfants
}

\author{
Jean-Matthieu Guillot, Marie-Alix Fauroux, Jean-Luc Pons, \\ Marie-Christine Goldsmith, Jacques-Henri Torres
}

Faculté d'Odontologie, 545 avenue Pr JL Viala, 34193 Montpellier Cedex 5, France

jeanmatthieuguillot@gmail.com

Actuellement en plein essor, la sédation consciente par mélange équimolaire oxygène-protoxyde d'azote (MEOPA) permet de prendre en charge davantage de patients au fauteuil et donc de limiter le nombre des anesthésies générales, notamment chez les enfants (Vaysse et al. 2004). Ayant fait la remarque empirique que les résultats de cette technique étaient moins bons à la fin d'une bouteille de gaz (en comparaison à une bouteille neuve), les auteurs ont souhaité étudier l'effet de la pression du gaz sur l'efficacité de la sédation.

Une enquête transversale a été menée au Service d'Odontologie sur une période de 5 mois. Elle a concerné les cas consécutifs de jeunes patients nécessitant une première séance de MEOPA pour des soins dentaires. Au total, 71 séances ont été menées sur des enfants de 2 à 14 ans $(37$ garçons, 34 filles). Plusieurs paramètres ont été renseignés concernant l'enfant et la séance de MEOPA (notamment, pression du gaz dans la bouteille en début et en fin de séance). Au cours des séances, ont été pratiqués des traitements conservateurs et/ou des avulsions dentaires. Les soins ont pu être menés à bien dans 64 cas sur les 71 (90\%). Parmi les 7 échecs, 3 sont survenus lors de l'anesthésie locale, 2 lors de l'utilisation de la turbine, 1 enfant a refusé le port du masque (et 1 échec non renseigné). Aucune différence significative n'a été notée entre les patients prémédiqués ou non-prémédiqués, ni entre les différentes classes d'âge. Une différence significative $(\mathrm{p}=0,0004)$ est apparue pour la pression initiale de la bouteille entre les cas de réussite $(139,19 \mathrm{KPa}$ en moyenne) et les cas d'échec $(71,17 \mathrm{KPa}$ en moyenne).

Le pourcentage de réussite obtenu dans cette étude est similaire aux résultats de la littérature (Droz et al. 2005, Collado et al. 2008, Fulgencio et al. 2004). Le résultat de cette étude sur l'influence de la pression du gaz au moment des soins corrobore l'expérience clinique des opérateurs. Cette relation n'avait semble-t-il pas fait l'objet de publication. L'effet de la pression sur l'efficacité de la sédation pourrait s'expliquer par une démixtion du mélange gazeux aux faibles pressions, entrainant une fraction inspirée par le patient contenant davantage d'oxygène et moins de protoxyde d'azote. Un autre élément doit également être pris en compte : la position de la bouteille. Dans le service, elle est en effet stockée et utilisée verticalement, alors que le fabriquant recommande une position horizontale, position susceptible de minimiser la démixtion gazeuse. En attendant le résultat d'une étude en cours destinée à mesurer l'effet de l'orientation de la bouteille et de la pression partielle des gaz sur la composition du mélange, il est conseillé de respecter le protocole d'utilisation du MEOPA et de vérifier la pression de bouteille. 